\title{
TOROIDAL MODELS OF MAGNETIC FIELD WITH TWISTED STRUCTURE
}

\author{
A.S. Petukhova \\ Yu.G. Shafer Institute of Cosmophysical Research \\ and Aeronomy SB RAS, \\ Yakutsk, Russia, petukhova@ikfia.ysn.ru
}

\author{
S.I. Petukhov \\ Yu.G. Shafer Institute of Cosmophysical Research \\ and Aeronomy SB RAS, \\ Yakutsk, Russia,i_van@ikfia.ysn.ru
}

\begin{abstract}
We present and discuss properties of the following magnetic field models in a magnetic cloud: Miller-Turner solution, modified Miller-Turner solution, Romashets-Vandas toroidal and integral models, and Krittinatham-Ruffolo model. Helicity of the magnetic field in all the models is the main feature of magnetic clouds. The first three models describe the magnetic field inside an ideal torus. In the integral model, parameters of a generating torus ambiguously determine the volume and shape of the magnetic field region. In the Krittinatham-Ruffolo model, the cross-section radius
\end{abstract}

of the torus is variable, thereby it corresponds more closely to the real form of magnetic clouds in the inner heliosphere. These models can be used to interpret insitu observations of the magnetic flux rope, to study a Forbush decrease in magnetic clouds and transport effects of solar energetic particles injected into a coronal mass ejection.

Keywords: magnetic flux rope models, force-free magnetic field, magnetic field lines, toroidal magnetic field, magnetic cloud.

\section{INTRODUCTION}

Ejections of coronal solar matter in interplanetary space are called ICMEs (interplanetary coronal mass ejections). Along with the matter, solar magnetic loops emerge which have a twisted structure of magnetic field lines - MFR (magnetic flux rope). Scientific interest in the study of MFR is due to the fact that MFR: 1) determines properties of the surrounding plasma; 2) greatly affects propagation of solar (SCR) and galactic cosmic rays (GCR) in interplanetary space; 3 ) determines the level of geomagnetic activity during the interaction with the magnetosphere. The practical interest in the MFR study is explained by the fact that SCR, GCR, and geomagnetic activity determine space weather conditions, which affect the safe operation of equipment and technological systems, as well as human activity. MFR with solar matter in ICME is called magnetic cloud (MC). MC occupies the total volume of ICME or its significant part [Marubashi, Lepping, 2007]. To study the processes occurring in MCs, it is necessary to use an MFR model. At present there are several MFR models with different features. The most widely used method of detecting MCs is based on the comparison of direct measurements of magnetic field components with the MFR model [Burlaga, 1988, Lepping et al., 1990, Farrugia et al., 1993, Leitner et al., 2007, Démoulin et al., 2008], which determines results. For example, the MC parameters obtained from the analysis of direct measurements with the use of cylindrical and toroidal MFR models differ significantly [Marubashi, Lepping, 2007]: 1) in the axis orientation of the magnetic field; 2) in cross-section radii (the radius in the toroidal model is smaller). Other authors employ different MFR models for studying GCR propagation in MCs: cylindrical [Kuwabara et al., 2004], toroidal [Petuhova et al., 2015].

In this paper, we present and compare features of five MFR models.

\section{FORCE-FREE MAGNETIC FIELD MODEL}

As an MFR model the force-free magnetic field is often utilized. The force-free magnetic field satisfies the relation

$$
\vec{j} \times \vec{B}=0
$$

where $\vec{j}$ is the electric current density, $\vec{B}$ is the magnetic field strength. Hence it follows that the current flows along the magnetic field $\vec{j} \sim \vec{B}$. In view of Maxwell's equations, the system of equations determining the force-free magnetic field can be written as

$$
\vec{\nabla} \times \vec{B}=\alpha \vec{B}, \vec{\nabla} \vec{B}=0
$$

where $\alpha$ is a scalar. When $\alpha$ is a constant or depends on coordinates, the magnetic field is called linear or nonlinear respectively. The second equation in (1) takes into account the solenoidal condition.

In real MCs, it is difficult to identify the force-free character of the magnetic field. In theoretical models, the similarity of the magnetic field to the force-free one is determined from the angle between $\vec{B}$ and $\vec{\nabla} \times \vec{B}$; in the force-free field this angle is zero [Vandas, Romashets, 2015].

\section{LUNDQUIST SOLUTION}

A solution of system of equations (1) for an infinitely extended cylinder has been put forward by Lundquist [Lundquist, 1950]. Components of the linear force-free field in the cylindrical coordinate system have the form

$$
B_{\rho}=0, B_{\theta}=-A J_{1}, B_{z}=A J_{0}
$$

where $J_{0}, J_{1}$ are the Bessel functions of the first kind of zero and first orders; $A= \pm B_{0}$, where $B_{0}$ is the field strength on the cylinder axis; $\alpha \rho$ is the argument of the 
Bessel functions; $\rho$ is the distance from the cylinder axis; $\alpha= \pm 2.41 / \rho_{0}$, where $\rho_{0}$ is the radius of cylinder cross-section. The solution accounts for the boundary condition $B_{z}\left(\rho_{0}\right)=0$, which is obtained taking into account $J_{0}(2.41)=0-$ the first root of $J_{0}$. The frame of the cylindrical coordinate system satisfies the vector product $\overrightarrow{i_{\theta}} \times \vec{i}_{\rho}=\vec{i}_{z}$, where $\vec{i}_{\theta, \rho, z}$ are unit vectors. The Lundquist solution includes four variants of magnetic field geometry: to each value of $B_{z}$ corresponds left- or right-handed helicity. On the surface of each cylinder with $\rho \leq \rho_{0}$ there are twisted magnetic field lines with their own step. The step changes from infinite on the cylinder axis to zero on its surface. The field strength decreases monotonically by a factor of two in the direction from the cylinder axis to the surface.

\section{MILLER-TURNER SOLUTION}

Miller and Turner calculated the magnetic field in a torus [Miller, Turner, 1981]. They used the quasitoroidal coordinate system related to Cartesian coordinates as follows

$$
\begin{aligned}
& x=(R+\rho \cos \theta) \cos \varphi, \\
& y=(R+\rho \cos \theta) \sin \varphi, \\
& z=\rho \sin \theta,
\end{aligned}
$$

where $R$ is the torus axis radius located in the XOY plane; $\rho$ is the distance from the torus axis in the perpendicular plane, $0 \leq \rho \leq \rho_{0}$, where $\rho_{0}$ is the cross-section radius of the torus; $\theta$ is the angle in this plane counted from XOY toward the Z-axis $(0 \leq \theta \leq 2 \pi) ; \varphi$ is the angle of this plane counted from the $\mathrm{X}$-axis toward the $\mathrm{Y}$-axis $(0 \leq \varphi \leq 2 \pi)$. The center of the Cartesian coordinate system coincides with the center of the torus. The frame of the quasi-toroidal coordinate system satisfies the vector product

$$
\overrightarrow{i_{\theta}} \times \overrightarrow{i_{\rho}}=\overrightarrow{i_{\varphi}}
$$

where $\vec{i}_{\rho, \varphi, \theta}$ are unit vectors. The choice of the frame has an effect on the form of the $\vec{\nabla}$ operator in the first equation of (1).

When accounting for the symmetry along the torus axis $(\partial / \partial \varphi=0)$, we can write the solution as

$$
\begin{aligned}
& B_{\rho}=\frac{A}{2 \alpha R} J_{0} \sin \theta \\
& B_{\theta}=-A\left[J_{1}-\frac{1}{2 \alpha R}\left(J_{0}+\alpha \rho J_{1}\right) \cos \theta\right], \\
& B_{\varphi}=A\left(1-\frac{\rho \cos \theta}{2 R}\right) J_{0} .
\end{aligned}
$$

Notations in (3) coincide with those in the Lundquist solution. When $R \rightarrow \infty$, (3) and Lundquist solutions coincide. Solution (3) is approximately force-free (it is supposed that $\rho_{0} / R<<1$ ) and satisfies the equation

$$
\vec{\nabla} \times \vec{B}=\alpha \vec{B}+\vec{H},
$$

where $\vec{H}$ is the discrepancy. It can be calculated by substituting solution (3) in the equation. This yields
$\vec{H}=-1.5\left(A J_{0} \rho \cos \theta\left(\overrightarrow{i_{\rho}} \sin \theta+\overrightarrow{i_{\theta}} \cos \theta\right) / R(R+\rho \cos \theta)\right)$. Solution (3) approximately satisfies the solenoidal condition

$$
\vec{\nabla} \vec{B}=3 A J_{1} \rho \sin \theta \cos \theta / 2 R(R+\rho \cos \theta) .
$$

Using relations (2), we obtain the metric coefficients $h_{\rho}=1, h_{\theta}=\rho, h_{\varphi}=R+\rho \cos \theta$.

To represent different magnetic field projections either when using magnetic field components in calculations or when comparing MFR with measurements, we have to use the solutions in different coordinate systems. To determine the magnetic field components in a desired system, we should identify them in the Cartesian coordinate system because it connects different systems. To calculate the magnetic field components in the Cartesian system, it is necessary to use the relationship between the components in different systems. Represent the linear vector $\overrightarrow{d r}$ in two systems

$$
\begin{aligned}
& \overrightarrow{d r}=\overrightarrow{i_{x}} d x+\overrightarrow{i_{y}} d y+\overrightarrow{i_{z}} d z=\overrightarrow{i_{\rho}} h_{\rho} d \rho+ \\
& +\overrightarrow{i_{\varphi}} h_{\varphi} d \varphi+\overrightarrow{i_{\theta}} h_{\theta} d \theta .
\end{aligned}
$$

Compute the differentials $d x, d y, d z$ through $d \rho, d \varphi, d \theta$ by using (2), and substitute them in the equation. Taking into account the independence of the differentials, obtain

$$
\begin{aligned}
& \overrightarrow{i_{\rho}}=\overrightarrow{i_{x}} \cos \theta \cos \varphi+\overrightarrow{i_{y}} \cos \theta \sin \varphi+\overrightarrow{i_{z}} \sin \theta, \\
& \overrightarrow{i_{\varphi}}=-\overrightarrow{i_{x}} \sin \varphi+\overrightarrow{i_{y}} \cos \varphi, \\
& \overrightarrow{i_{\theta}}=-\overrightarrow{i_{x}} \sin \theta \cos \varphi-\overrightarrow{i_{y}} \sin \theta \sin \varphi+\overrightarrow{i_{z}} \cos \theta .
\end{aligned}
$$

Represent the vector for two coordinate systems:

$$
\vec{B}=\overrightarrow{i_{x}} B_{x}+\overrightarrow{i_{y}} B_{y}+\overrightarrow{i_{z}} B_{z}=\overrightarrow{i_{\rho}} B_{\rho}+\overrightarrow{i_{\theta}} B_{\theta}+\overrightarrow{i_{\varphi}} B_{\varphi}
$$

and, using (4), get

$$
\begin{aligned}
& B_{x}=B_{\rho} \cos \theta \cos \varphi-B_{\varphi} \sin \varphi-B_{\theta} \sin \theta \cos \varphi, \\
& B_{y}=B_{\rho} \cos \theta \sin \varphi+B_{\varphi} \cos \varphi-B_{\theta} \sin \theta \sin \varphi, \\
& B_{z}=B_{\rho} \sin \theta+B_{\theta} \cos \theta .
\end{aligned}
$$

To determine the shape of the magnetic field line, use the definition $\overrightarrow{d l}=\vec{B} d l / B$. Here $\overrightarrow{d l}$, $d l$ are the vector and length of the field line element; $\vec{B}$ and $B$ are the magnetic field vector and strength. From the definition it follows:

$$
\begin{aligned}
& d \rho=B_{\rho} d l / h_{\rho} B, \\
& d \theta=B_{\theta} d l / h_{\theta} B, \\
& d \varphi=B_{\varphi} d l / h_{\varphi} B .
\end{aligned}
$$

We plot the magnetic field line according to the recurrent procedure: select $(\rho, \theta, \varphi)$; compute $B_{\rho}, B_{\theta}, B_{\varphi}$ according to (3); calculate $d \rho, d \theta, d \varphi$ according to (6); at a new point $\rho+d \rho, \theta+d \theta, \varphi+d \varphi$ calculate $B_{\rho}, B_{\theta}, B_{\varphi}$, etc. To determine the magnetic field line in the Cartesian coordinate system, use (2) and (5).

Figure $1, a, b$ shows the magnetic field parameters for the Miller-Turner solution. Figure 1, $a$ presents the distri- 

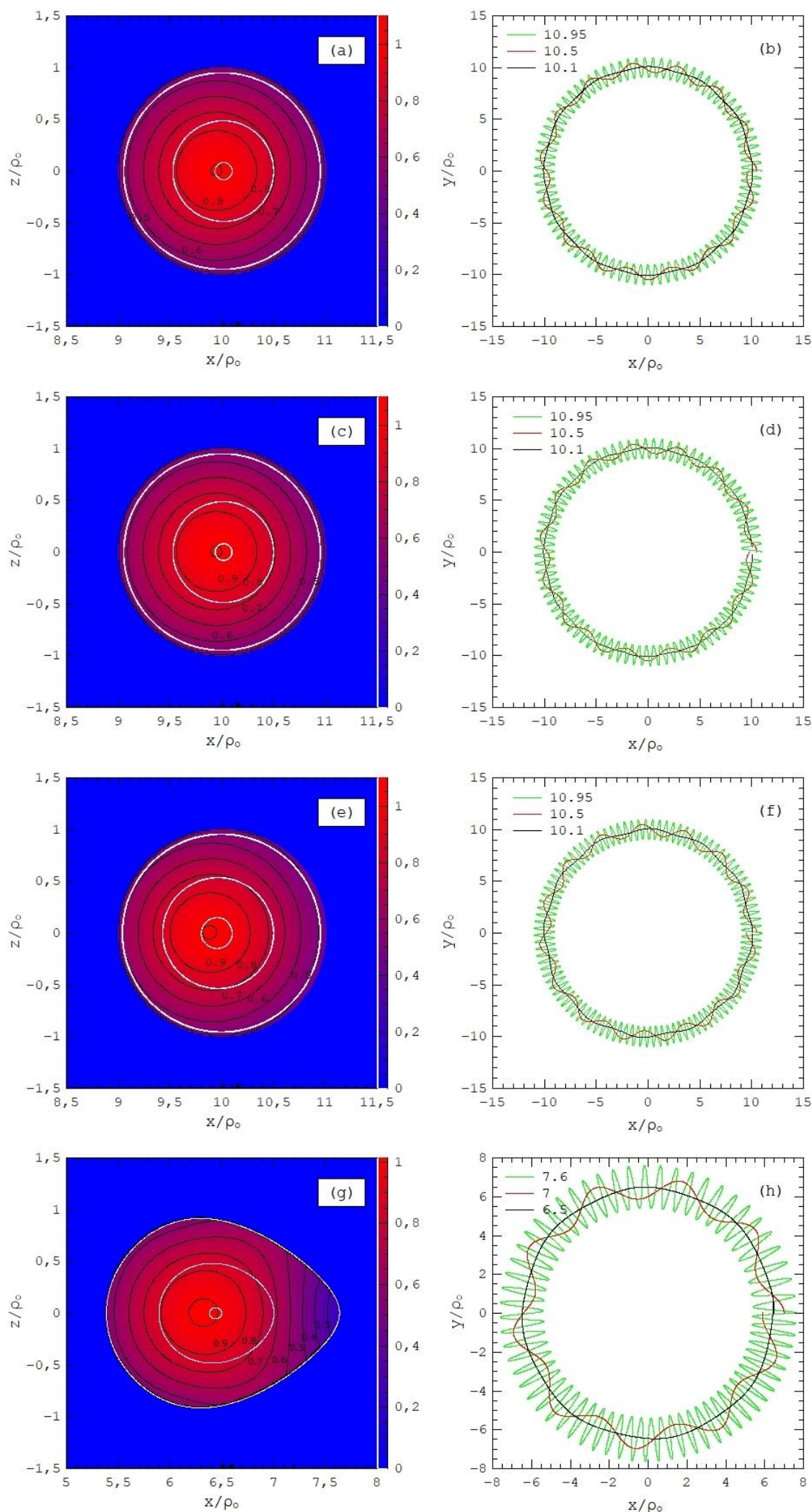

Figure 1. Distribution of the relative magnetic field strength $\left(B / B_{0}\right)$ in the plane perpendicular to the torus axis in the Miller-Turner solution $(a)$, modified Miller-Turner solution $(c)$, toroidal $(e)$ and integral $(g)$ Romashets-Vandas solutions respectively. Color indicates magnetic field strength according to the scale shown on the right-hand side of the Figures. Black curves and numbers near them are isolines of the magnetic field strength. White curves are field line projections for three variants differing in $x$ coordinate of the starting point of the field line. For panels $a, c, e$, the coordinates of the starting point of field lines are $y=0, z=0, x / \rho_{0}=10.95,10.5,10.1$. For panels $g, h$, the coordinates of the starting point of field lines are $y=0, z=0, x / \rho_{0}=7.6,7,6.5$. Panels $b, d, f, h$ show projections of magnetic force lines on the XOY plane for the same three variants. The variants differ in color of magnetic field lines, shown in the top left corner 
butions of the relative field strength $\left(B / B_{0}\right)$ in the plane of the torus cross-section and the projections of the field lines, located on the surface of toroids with different radii, on this plane. Figure $1, b$ shows the projections of the field lines, located on the surface of different toroids, on the XOY plane. Here, we utilize the toroidal surfaces with radii $\rho_{\mathrm{T}}<\rho_{0}$, located inside the torus, as toroids. The field lines are seen to be on the surface of each toroid. In this case, the field lines are represented as helices with a step depending on the toroid radius: the smaller the radius, the larger the step. These features are typical of the force-free magnetic field. Figure 1, $a$ shows that the field axis is slightly shifted from the center of the cross section in the direction from the center of the torus. At the same time, the maximum field strength is shifted toward the center of the torus. The ratio between the magnetic field strengths on the torus axis and surface is limited by 2 . The calculation results presented in Figure 1, $a-f$ are obtained for $R / \rho_{0}=10, A=B_{0}, \alpha=$ $2.41 / \rho_{0}$. There are four variants of the magnetic field geometry as in the Lundquist solution. The magnetic field variants differ in sign of the field components; however, this difference has no effect on the field line projections depicted in Figure 1, $a-f$.

\section{MODIFIED MILLER-TURNER SOLUTION}

Romashets and Vandas [2003b] have modified the Miller-Turner solution. They used the vector potential of the modified solution

$$
\vec{A}^{(m)}=\frac{1}{\alpha} \vec{B},
$$

where $\vec{B}$ is Miller-Turner solution (3). Then

$$
\vec{B}^{(m)}=\vec{\nabla} \times \vec{A}^{(m)}=\frac{1}{\alpha} \vec{\nabla} \times \vec{B}=\frac{1}{\alpha}(\alpha \vec{B}+\vec{H})=\vec{B}+\frac{\vec{H}}{\alpha},
$$

where $\vec{B}^{(m)}$ is the strength of the modified magnetic field, $\vec{H}$ is the discrepancy of the Miller-Turner solution. Using solution (3), obtain components of the modified magnetic field

$$
\begin{aligned}
& B_{\rho}^{(m)}=\frac{A J_{0} \sin \theta}{2 \alpha R} \frac{(R-2 \rho \cos \theta)}{(R+\rho \cos \theta)}, \\
& B_{\theta}^{(m)}=\frac{A}{2 \alpha R(R+\rho \cos \theta)}\left[2 \alpha R^{2} J_{1}-R \cos \theta \times\right. \\
& \left.\times\left(J_{0}-\alpha \rho J_{1}\right)+\rho\left(2 J_{0}-\alpha \rho J_{1}\right) \cos ^{2} \theta\right], \\
& B_{\varphi}^{(m)}=A J_{0}(1-\rho \cos \theta /(2 R)) .
\end{aligned}
$$

Notations of the values coincide with those in (3). The modified solution is also approximate $\left(\rho_{0} / R<<1\right)$, and exactly satisfies the solenoidal condition. The construction of magnetic field lines is similar to that in the Miller-Turner solution.

Figure $1, c, d$ similar to Figure $1, a, b$ presents magnetic field parameters for the modified Miller-Turner solution. The comparison between these Figures suggests that the magnetic field parameters in the Miller-Turner solution and in their modified solution differ slightly.

\section{ROMASHETS-VANDAS SOLUTION}

To calculate the magnetic field in the torus, Romashets and Vandas [2003a] used the toroidal system of coordinates related to Cartesian coordinates as

$$
\begin{aligned}
& x=\frac{\alpha \sinh (\mu) \cos \varphi}{\cosh (\mu)-\cos \eta}, \\
& y=\frac{\alpha \sinh (\mu) \sin \varphi}{\cosh (\mu)-\cos \eta}, \\
& z=\frac{\alpha \sin \eta}{\cosh (\mu)-\cos \eta} .
\end{aligned}
$$

Here $\alpha$ is the coordinate system parameter specified by the size of a chosen torus $\rho_{0}=\alpha / \sinh \left(\mu_{0}\right)$, $R=\alpha \cosh \left(\mu_{0}\right) / \sin h\left(\mu_{0}\right), \quad R / \rho_{0}=\cosh \left(\mu_{0}\right), \quad \alpha=\sqrt{R^{2}-\rho_{0}^{2}}=$ $=\rho_{0} \sqrt{\left(R / \rho_{0}\right)^{2}-1}$, where $\rho_{0}$ is the radius of the torus cross-section; $R$ is the distance from the torus center to the axis; $\sinh (\mu), \cosh (\mu)$ are hyperbolic sine and cosine. The parameters are determined by $\mu \geq \mu_{0}, 0 \leq \eta \leq 2 \pi$, $0 \leq \varphi \leq 2 \pi$, where $\mu_{0}$ corresponds to the surface of a chosen torus.

The solution of the problem can be represented as follows

$$
\begin{aligned}
& B_{\mu}=0, \\
& B_{\eta}=-A \frac{\varepsilon \cosh (\mu)(\cosh (\mu)-\cos \eta)}{2 \sinh ^{3}(\mu)} F_{1}, \\
& B_{\varphi}=A \frac{\cosh (\mu)-\cos \eta}{\sinh (\mu)} F_{0},
\end{aligned}
$$

where $F_{0}=F\left(\alpha_{0}, \beta_{0}, \gamma_{0}, \xi\right), F_{1}=F\left(1+\alpha_{0}, 1+\beta_{0}, 1+\gamma_{0}, \xi\right)$ are hypergeometric functions, $\quad \alpha_{0}=\left(1+\sqrt{1-4 \varepsilon^{2}}\right) / 4$, $\beta_{0}=\left(1-\sqrt{1-4 \varepsilon^{2}}\right) / 4, \gamma_{0}=1, \xi=-\sinh ^{-2}(\mu)$.

In the toroidal coordinate system, all surfaces with $\mu=$ const are coordinate surfaces (toroids). The surface with $\mu=\mu_{0}$ coincides with the surface of the given torus, and surfaces with $\mu>\mu_{0}$ describe the toroids located inside the torus. The surface with $\mu \rightarrow \infty$ degenerates into the torus axis. Solution (9) represents a magnetic field with two components whose field lines are located on the surface of the toroids. The $\varepsilon$ value is determined from $F_{0}\left(\alpha_{0}, \beta_{0}, \gamma_{0},-\sinh ^{-2}\left(\mu_{0}\right)\right)=0$. As follows from (9), in this case $B_{\varphi}=0$ on the torus surface. This condition is similar to the use of the first root of $J_{0}(2.41)=0$ in the Lundquist solution.

The ratios between the field components in the Cartesian and toroidal systems are derived from

$$
\vec{B}=\overrightarrow{i_{x}} B_{x}+\overrightarrow{i_{y}} B_{y}+\overrightarrow{i_{z}} B_{z}=\overrightarrow{i_{\eta}} B_{\eta}+\overrightarrow{i_{\varphi}} B_{\varphi},
$$

which takes into account that the magnetic field in the toroidal system has two components $\left(B_{\mu}=0\right)$. Unit vectors of the coordinate systems are related as 


$$
\begin{aligned}
& \overrightarrow{i_{\eta}}=\frac{1}{h_{\eta}}\left(\overrightarrow{i_{x}} \frac{\partial x}{\partial \eta}+\overrightarrow{i_{y}} \frac{\partial y}{\partial \eta}+\overrightarrow{i_{z}} \frac{\partial z}{\partial \eta}\right), \\
& \overrightarrow{i_{\varphi}}=\frac{1}{h_{\varphi}}\left(\overrightarrow{i_{x}} \frac{\partial x}{\partial \varphi}+\overrightarrow{i_{y}} \frac{\partial y}{\partial \varphi}\right),
\end{aligned}
$$

where $h_{\eta}=\alpha /(\cosh (\mu)-\cos \eta), h_{\varphi}=\alpha \sinh (\mu) /(\cosh (\mu)-\cos \eta)$ are metric coefficients. Calculate the partial derivatives, using (8). This yields

$$
\begin{aligned}
& B_{x}=B_{\eta} \sinh (\mu) \sin \eta \cos \varphi /(\cosh (\mu)-\cos \eta)- \\
& -B_{\varphi} \sin \varphi \\
& B_{y}=B_{\eta} \sinh (\mu) \sin \eta \sin \varphi /(\cosh (\mu)- \\
& -\cos \eta)+B_{\varphi} \cos \varphi, \\
& B_{z}=B_{\eta}(\cosh (\mu) \cos \eta-1) /(\cosh (\mu)-\cos \eta) .
\end{aligned}
$$

Determine magnetic field lines from the equation

$$
d l=\overrightarrow{i_{x}} d x+\overrightarrow{i_{y}} d y+\overrightarrow{i_{z}} d z=\left(\overrightarrow{i_{x}} B_{x}+\overrightarrow{i_{y}} B_{y}+\overrightarrow{i_{z}} B_{z}\right) d l / B,
$$

where $d l=\sqrt{d x^{2}+d y^{2}+d z^{2}}, B=\sqrt{B_{x}^{2}+B_{y}^{2}+B_{z}^{2}}$.

Figure $1, e, f$ shows the same as Figure $1, a, b$. The region of the maximum magnetic field strength is seen to be shifted toward the torus center. The magnetic field strength in the interior is limited by 10 .

\section{INTEGRAI MODEL}

Romashets and Vandas have developed a toroidal field model [Romashets, Vandas, 2009], using the Lundquist solution. They introduced a generating torus with the cross-section radius $\rho_{0}$ and the torus axis radius $R$. The torus axis is located in the XOY plane of the laboratory Cartesian coordinate system, and the torus center coincides with the system center. The authors added auxiliary cylinders with the crosssection radius $\rho_{0}$ whose axes are in the XOY plane and are tangent to a circle of radius $R$, whose center coincides with the torus center (torus axis). The magnetic field inside and outside the cylinder is the Lundquist solution.

The toroidal field is formed by the superposition of magnetic fields of the auxiliary cylinders with the angular size $d \varphi$ :

$$
\begin{aligned}
& B_{x}=A \int_{0}^{2 \pi}\left(J_{1} z \cos \varphi / \rho-J_{0} \sin \varphi\right) d \varphi, \\
& B_{y}=A \int_{0}^{2 \pi}\left(J_{1} z \sin \varphi / \rho+J_{0} \cos \varphi\right) d \varphi, \\
& B_{z}=-A \int_{0}^{2 \pi}\left(J_{1}(x \cos \varphi+y \sin \varphi-R) / \rho\right) d \varphi,
\end{aligned}
$$

where $\rho=\sqrt{z^{2}+(x \cos \varphi+y \sin \varphi-R)^{2}}$.

The Lundquist solution describes a force-free magnetic field with a constant $\alpha$; all fields of auxiliary cylinders are force-free fields with the same value $\alpha$, hence field superposition (11) is also a force-free field with the same value $\alpha$.

The parameters are shown in Figure $1, g, h$ for $R=6$, $\alpha=2.41, \rho_{0}=1$. The magnetic field is seen to be located outside the generating torus (maximum and minimum radii of the torus relative to its center are 7 and 5 respec- tively); the projections of the field lines on the crosssection differ markedly from the circular ones. The magnetic field strength is limited by 5 . Parameters of the generating torus do not uniquely determine the shape and volume of the region occupied by the magnetic field. At other distances, field components (11) are the magnetic fields differing from that shown in Figure $1, g, h$ in volume and shape of the region.

\section{KRITTINATHAM-RUFFOLO MODEL}

Krittinatham and Ruffolo put forward an analytical model of the magnetic field in a loop representing MC [Krittinatham, Ruffolo, 2009].

We use the quasi-toroidal coordinate system as in Miller-Turner model (2). In the model calculation, we assume that $R=0.5 r_{\mathrm{e}}, \rho_{0}=0.1 r_{\mathrm{e}}$, where $r_{\mathrm{e}}$ is the astronomical unit. In this case, the axis with $\varphi=\pi, x / r_{\mathrm{e}}=-0.5$ passes through the Sun; and with $\varphi=0, x / r_{\mathrm{e}}=0.5$, through Earth. The shape of the magnetic field lines is given by the relations

$$
\begin{aligned}
& \rho=d \cos (\varphi / 2), \\
& \theta=w \pi e^{-d / d_{0}} \sin (\varphi / 2)+\theta_{0},
\end{aligned}
$$

where $0 \leq \varphi \leq 2 \pi, 0 \leq \theta \leq 2 \pi, 0 \leq d \leq \rho_{0} 2 \pi$. Each field line is determined by $d, \theta_{0}$ when $\varphi=0$. From the first relation of (12) it follows that the cross-section area of the loop tends to zero at the approach to the Sun. The value $w$ is the total number of complete circuits of the field line at the angle $\theta$ located near the axis (magnetic helicity). The sign of $w$ specifies the sign of the magnetic helicity: when $w>0$, the helicity is negative and vice versa. The exponential factor in the second equation of (12) through $d_{0}$ takes into account the change in the helicity with distance from the axis. The construction of field lines is the same as in the Miller-Turner model. In view of (12), calculate the differentials $d \rho=-(d / 2) \sin (\varphi / 2) d \varphi$, $d \theta=(w \pi / 2) e^{-d / d_{0}} \cos (\varphi / 2) d \varphi$, used in the recurrent procedure. To calculate magnetic field components, take into account the relationship between the shape of field lines and the components

$$
\begin{aligned}
& B_{\rho}=\left(B_{\varphi} / h_{\varphi}\right) d \rho / d \varphi, \\
& B_{\theta}=\left(B_{\varphi} h_{\theta} / h_{\varphi}\right) d \theta / d \varphi, \\
& h \rho=1 . \\
& \text { For } B_{\varphi}, \text { we utilize the equation } \\
& B_{\varphi}=B_{0} /\left[\left(d^{2} / d_{0}^{2}+1\right)^{3 / 2} \cos ^{2}(\varphi / 2)\right],
\end{aligned}
$$

which accounts for preservation of the magnetic flux through the cross-section of the loop. Considering the first relation from (12), we get

$$
\begin{aligned}
& B_{\rho}=-\frac{B_{0}}{2} \sin \left(\frac{\varphi}{2}\right) F \frac{\rho}{R+\rho \cos \theta}, \\
& B_{\varphi}=B_{0} \cos \left(\frac{\varphi}{2}\right) F, \\
& B_{\theta}=\frac{B_{0} w \pi}{2} \cos ^{2}\left(\frac{\varphi}{2}\right) F \frac{\rho}{R+\rho \cos \theta} e^{-\rho /\left[d_{0} \cos (\varphi / 2]\right.},
\end{aligned}
$$

where $F=\left[\rho^{2} / d_{0}^{2}+\cos ^{2}(\varphi / 2)\right]^{-3 / 2}$. 
Figure 2, $a, c$ shows distributions of the magnetic field strength in the plane perpendicular to the axis. Field line projections are circles with the center coinciding with the axis. Figure $2 b, d$ depicts projections of three field lines, located at different distances from the axis, on the XOY plane. In the calculation, we use $R=0.5 r_{\mathrm{e}}, \rho_{0}=0.1 r_{\mathrm{e}}, w=8, d_{0}=0.07$. Referring to Figure 2, $a, b$, the magnetic field strength distribution corresponds to the force-free one, whereas the helicity distribution does not. If we change the sign of the exponential factor in the second equation of (12), maxima of magnetic field strength and magnetic helicity will be on the loop surface (Figure 2, $c, d$ ).

\section{CONCLUSION}

The approximate Miller-Turner solution $\left(\rho_{0} / R<<1\right)$ describes the magnetic field structure in the torus. The structure may have four forms: negative and positive helicities in different directions along the torus axis. Properties of the magnetic field correspond to those in the cylindrical Lundquist model. The magnetic field strength is maximum on the torus axis and twice as high as that on the surface. The magnetic helicity is maximum on the torus surface. The modified Miller-Turner solution exactly satisfies the solenoidal condition. The magnetic field properties in the Miller-Turner solution
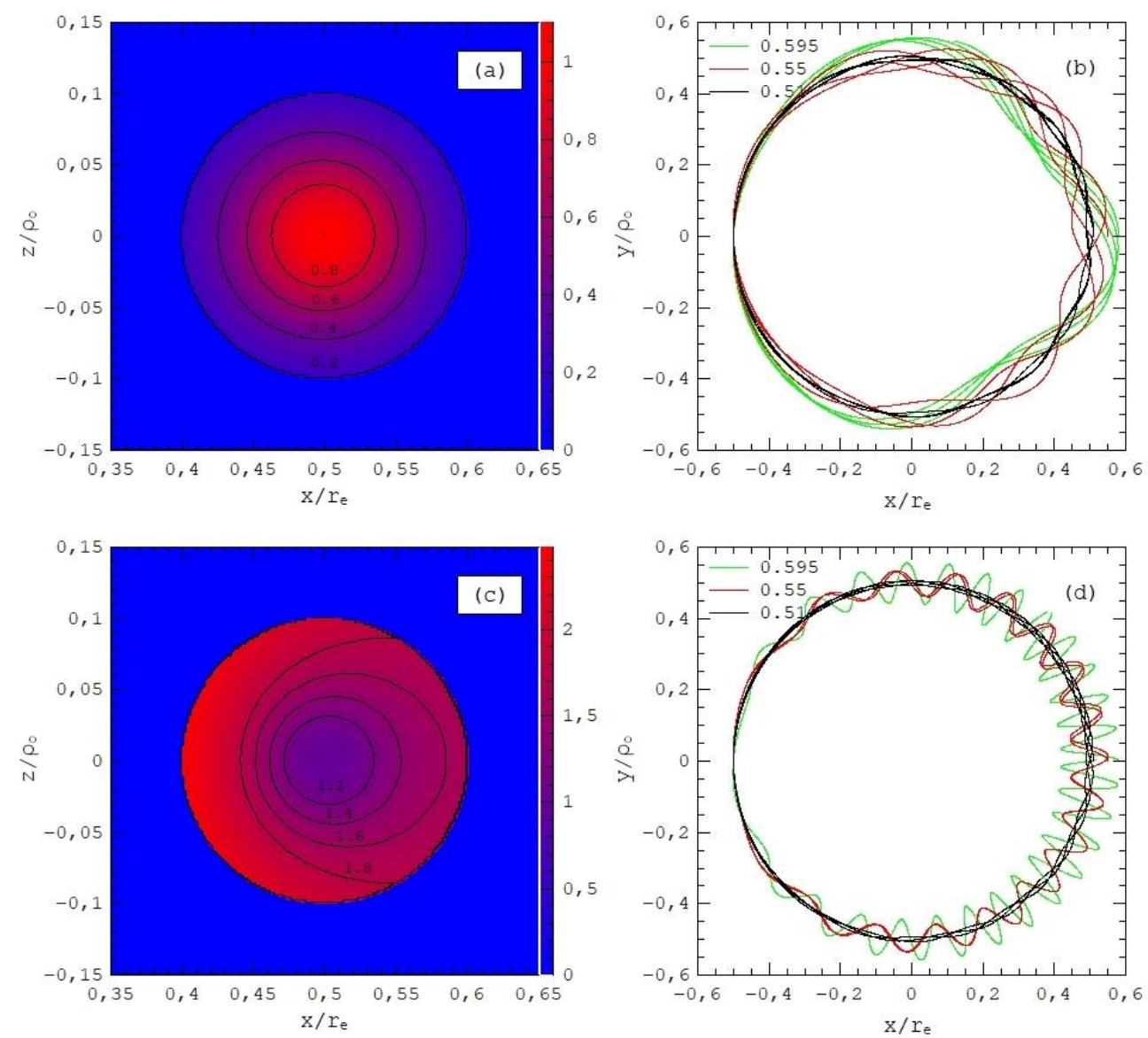

differ slightly from those in the modified Miller-Turner solution. We have established that the approximate Miller-Turner solution corresponds quite closely to the exact solution for the force-free field in the torus up to $\rho_{0} / R \leq 0.5$ [Vandas, Romashets, 2015].

A substantially asymmetric distribution of magnetic field properties is obtained in the toroidal Romashets and Vandas model. The asymmetry of the distribution strongly depends on $R / \rho_{0}$ : it increases when $\mathrm{R} / \rho 0$ decreases. In the integral model, the resulting magnetic field is outside the generating torus; the magnetic field strength distribution is highly inhomogeneous. Parameters of the generating torus do not uniquely determine the volume and shape of the region occupied by the magnetic field. At other distances, field components (11) are the magnetic fields differing from that shown in Figure $1, g, h$ in volume and shape of the region. The Krittinatham-Ruffolo analytical model presents magnetic field components in the loop representing a magnetic cloud. The cross-section area of the loop tends to zero when the loop approaches the Sun. Magnetic field lines have left or right-handed helicity. The magnetic field strength and magnetic helicity decrease in the direction from the axis of the loop to its surface.

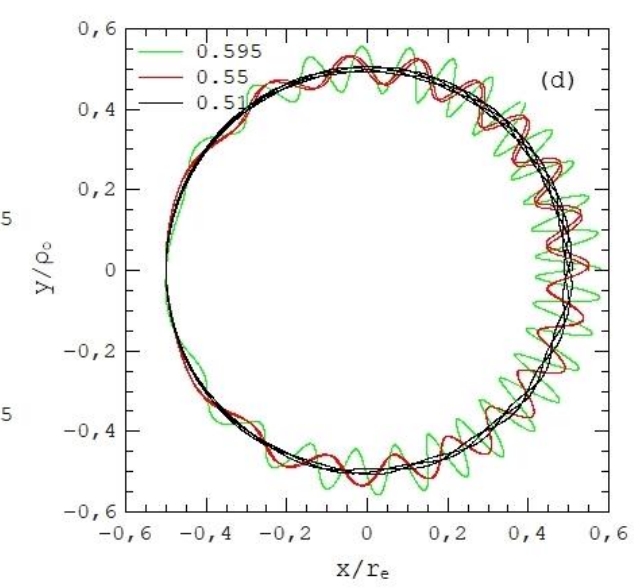

Figure 2. Distribution of the relative magnetic field strength $\left(B / B_{0}\right)$ in the plane perpendicular to the loop axis $(a, c)$. Color indicates magnetic field strength according to the scale shown on the right-hand side. Black curves and numbers near them are isolines of the magnetic field strength. Panels $b, d$ present projections of magnetic field lines on the XOY plane for three field lines differing in $x$ coordinate of the starting point of the field line $y=0, z=0, x / r_{\mathrm{e}}=0.595,0.55,0.51$ 
The Miller-Turner and Romashets-Vandas toroidal models are better suited for description of magnetic fields in MCs.

The implemented program codes for the five magnetic field models with explanations are available on [https://github.com/ivanpetukhov1978/Models-of-magneticfield/releases/tag/1.0], DOI: 10.5281/zenodo.1728477. When using the codes, reference should be made to this paper.

This work was supported by the Russian Foundation for Basic Research (grant No. 18-32-0064 mol_a), the Ministry of Education and Science of the Russian Federation, and the Siberian Branch of the Russian Academy of Sciences (Project II.16.2.2.).

\section{REFERENCES}

Burlaga L.F. Magnetic clouds and force-free fields with constant alpha. J. Geophys. Res. 1988, vol. 93, no. A7, pp. 72177224. DOI: 10.1029/JA093iA07p07217.

Démoulin P., Nakwacki M.S., Dasso S., Mandrini C.H. Expected in situ velocities from a hierarchical model for expanding interplanetary coronal mass ejections. Solar Phys. 2008, vol. 250, no. 2, pp. 347-374. DOI: 10.1007/s11207-0089221-9.

Farrugia C.J., Burlaga L.F., Osherovich V.A., Richardson I.G., Freeman M.P., Lepping R.P., Lazarus A.J. A study of an expanding interplanetary magnetic cloud and its interaction with the Earth's magnetosphere: The interplanetary aspect. J. Geophys. Res. 1993, vol. 98, pp. 76217632. DOI: 10.1029/92JA02349.

Krittinatham W., Ruffolo D. Drift orbits of energetic particles in an interplanetary magnetic flux rope. Astrophys. J. 2009, vol. 704, pp. 831-841. DOI: 10.1088/0004-637X/704/1/831.

Kuwabara T., Munakata K., Yasue S., Kato C., Akahane S., Koyama M., Bieber J.W., et al. Geometry of an interplanetary CME on October 29, 2003 deduced from cosmic rays. Geophys. Res. Lett. 2004, vol. 31, p. L19803.

Leitner M., Farrugia C.J., Möstl C., Ogilvie K.W., Galvin A.B., Schwenn R., Biernat H.K. Consequences of the force-free model of magnetic clouds for their heliospheric evolution. J. Geophys. Res. 2007, p. 112. DOI: 10.1029/ 2006JA011940.

Lepping R.P., Jones J.A., Burlaga L.F. Magnetic field structure of interplanetary magnetic clouds at $1 \mathrm{AU}$. J. Geophys. Res. 1990, vol. 95, pp. 11957-11965. DOI: 10.1029/JA095iA 08p11957.

Lundquist S. Magnetohydrostatic fields. Ark. Fys. 1950, vol. 2, p. 361 .

Marubashi K., Lepping R.P. Long-duration magnetic clouds: a comparison of analyses using torus- and cylindershaped flux rope models. Ann. Geophys. 2007. V. 25, P. 2453 2477. DOI: $10.5194 /$ angeo-25-2453-2007.

Miller G., Turner L. Force free equilibria in toroidal geometry. Physics of Fluids. 1981, vol. 24, pp. 363-365. DOI: $10.1063 / 1.863351$.

Petukhova A.S., Petukhov I.S., Petukhov S.I. Forbush decrease in the intensity of cosmic rays in a toroidal model of a magnetic cloud. JETP Lett. 2015, vol. 102, pp. 697-700.

Romashets E.P., Vandas M. Force-free field inside a toroidal magnetic cloud. Geophys. Res. Lett. 2003a, vol. 30, pp. 2065-2069. DOI: 10.1029/2003GL017692.

Romashets E.P., Vandas M. Interplanetary magnetic clouds of toroidal shapes. Proc. of International Solar Cycle Studies (ISCS) 2003 Symposium. 2003b, pp. 535-540. DOI: $10.1051 / 0004-6361 / 200911701$.
Romashets E.P., Vandas M. Linear force-free field of a toroidal symmetry. Astron. Astrophys. 2009, vol. 499, pp. 17-20.

Vandas M., Romashets E.P. Comparative study of a constantalpha force-free field and its approximations in an ideal toroid. Astron. Astrophys. 2015, vol. 580, p. A123. DOI: 10.1051/00046361/201526242.

URL: https://github.com/ivanpetukhov1978/Models-ofmagnetic-field/releases/tag/1.0 (accessed March 1, 2019).

How to cite this article

Petukhova A.S., Petukhov S.I. Toroidal models of magnetic field with twisted structure. Solar-Terrestrial Physics. 2019. Vol. 5. Iss. 2. P. 69-75. DOI: 10.12737/stp-52201910. 